\title{
Effects of Prostaglandin E1 and Supplemental Oxygen on the Wound Healing
}

\author{
Bongsoo Baik (D), Sulki Park (D), Soyoung Ji (D), Wansuk Yang (D), Junekey Lee (D) \\ Department of Plastic and Reconstructive Surgery, Dongkang Medical Center, Ulsan, Korea
}

\begin{abstract}
Background: When an avulsion wound is combined with a crushing injury or when a local flap is moved with significant tension, poor local blood supply may result in partial or complete necrosis of the involved tissue. This paper explores procedures to prevent tissue necrosis for the ischemic wounds.

Methods: From March 2017 to December 2018, 29 hospitalized patients (group A) were treated with simple dressing change and administration of antibiotics. From January 2019 to October 2020, 29 hospitalized patients (group B) were injected for the first 3 days intravenously once a day with $50 \mu \mathrm{g}$ of stabilized prostaglandin E1. Prostaglandin E1 injections were combined with supplemental oxygen $(4 \mathrm{~L} / \mathrm{min}$ through nasal cannula for 24 hours per day for the first 3 days). Wound dressing and antibiotics administration were also combined.

Results: Ten patients in group A developed partial wound necrosis, out of which four patients received local flap surgery after excision of the necrotic tissue. The average recovery time was 9.7 days. In group B, four patients developed partial wound necrosis, out of which one was treated with local flap surgery. The average recovery for the four patients in group B took 6.2 days.

Conclusion: Prostaglandin E1 and supplemental oxygen reduced the incidence of partial wound necrosis of ischemic wounds and local flap surgery after excision of the necrotic tissue, and also shortened the average recovery time.
\end{abstract}

Keywords: Prostaglandin E1; Avulsion; Necrosis; Wound healing

\section{Introduction}

Most lacerated wounds faced in the emergency room are treated on an ambulatory care basis upon completion of skin sutures; however, severe wounds sustained from motor vehicle or industrial sites accidents, etc. are treated in inpatient care. The cases requiring inpatient care include irregular lacerations in tissues or crushing injuries with avulsion wounds that result in insufficient blood circulation in the injured tissues. There have been cases among inpatients wherein partial necrosis of sutured wounds occurred, for which debridement and revisional repair or application of local flap were conducted. Upon witnessing such cases, the authors explored the causes for unfavorable consequences and ways to ameliorate such consequences. Based on literature describing the application of prostaglandin E1 (PGE1, alprostadil) and supplemental oxygen to clinical practice with promising therapeutic outcomes, we decided to employ the following therapies.

PGE1 is a hormone-like substance, and the exact mechanism of action is not fully known. However, an experimental study demonstrated that it induces vasodilation and increases vascular flow [1]. It also has antiplatelet and fibrinolytic properties [2,3]. These mechanisms contribute to the anti-ischemic effects of PGE1 on flap survival [4]. The essential role of oxygen in wound healing may support vital processes such as angiogenesis, cell motility, and extracellular matrix formation [5].

\section{Original Article}

Received: March 29, 2021

Revised: May 14, 2021

Accepted: May 15, 2021

\section{Corresponding author:}

Sulki Park, M.D.

Department of Plastic and Reconstructive Surgery, Dongkang Medical Center, 39 Taehwaro, Jung-gu, Ulsan 44455, Korea

Tel: +82-52-241-1114

Fax: +82-52-241-1180

E-mail: jammting2@gmail.com

This is an Open Access article distributed under the terms of the Creative Commons Attribution Non-Commercia License (https://creativecommons org/licenses/by-nc/4.o/) which permits unrestricted non-commercial use, distribution, and reproduction in any medium, provided the original work is properly cited.

C) 2021 Korean Wound Management Society 
We therefore aimed to compare the therapeutic results of administrating PGE1 and supplemental oxygen to inpatients with traumatic wounds with a control group receiving conventional wound care.

\section{Methods}

A total of 5,425 patients came to the emergency room to receive emergency plastic surgical treatment for traumatic wounds during the 44-month period from March 2017 to October 2020. Among them, 118 patients were subjected to inpatient care. The 67 patients hospitalized from March 2017 to December 2018 were initially allocated to the control group (group A). Patients whose age and wound characteristics (location, size, depth, clean or contaminated status) matched those from group A were selected from the 51 patients hospitalized between January 2019 and August 2020 as the experimental group (group B). Through this matching process, 29 patients each were finally designated to groups A and B. This was to minimize other external factors besides PGE1 and supply of oxygen that might affect recovery of the wounds. The study was approved by the Institutional Review Board of Dongkang Medical Center (IRB No. 2021-01-01) and performed in accordance with the principles of the Declaration of Helsinki. Written informed consent was obtained upon admission of all patients included in the study. The proportion of patients of the age class likely to be exposed to outdoor activities or a risk-prone occupational environment was relatively dominant. Compared to wounds on other areas, facial wounds were most common, for which most patients were treated with skin suture or flap surgery before admission (Table 1).

Patients in group A were treated with simple dressing changes and antibiotics administration. Patients in group B were administered an intravenous injection of $50 \mu \mathrm{g}$ of Prostandin (Dong-A Pharm., Seoul, Korea) dissolved in $500 \mathrm{~mL}$ of normal saline once a day for the first 3 days. Prostandin is an alprostadil alfadex formulation developed as a stabilized analog of PGE1. In addition, group B patients were also treated with supplemental oxygen therapy $(4 \mathrm{~L} / \mathrm{min}$ through a nasal cannula for 24 hours per day for first 3 days). Wound dressings and antibiotics administration were the default treatment for both groups. Dressing was performed once a day in both groups, and the wounds were monitored according to standard flap monitoring. In checking the wound condition, the skin color of wound, temperature, and capillary refill time of each patient was watched, and the presence of necrosis was
Table 1. Demographics and characteristics of the patients

\begin{tabular}{lccc}
\hline Characteristics & Group A & Group B & Total \\
\hline Sex & & & \\
Male & 19 & 17 & $36(62.1)$ \\
Female & 10 & 12 & $22(37.9)$ \\
Age (yr) & & & \\
$18-40$ & 12 & 12 & $24(41.4)$ \\
$41-60$ & 14 & 14 & $28(48.3)$ \\
61-80 & 3 & 3 & $6(10.3)$ \\
Location & 27 & 27 & $54(93.2)$ \\
Face \& neck & 1 & 1 & $2(3.4)$ \\
Upper limbs & 1 & 1 & $2(3.4)$ \\
Lower limbs & & & \\
\hline
\end{tabular}

Values are presented as number or number (\%). Group $A$, the group of inpatients treated with simple dressings and antibiotics administration; Group B, the group of inpatients treated with simple dressings, antibiotics administration, prostaglandin $\mathrm{E} 1$ and supplemental oxygen for 3 days upon admission.

also observed.

As the study was conducted on a large number of hospitalized patients, it must be considered that differences in blood vessel resilience for patients of differing ages could have affected the outcome of the study. Therefore, patients were divided by age group and statistically analyzed for their association with age and partial necrosis using IBM SPSS Statistic version 26.0 (IBM Corp., Armonk, NY, USA). The association between the use of PGE1, supplemental oxygen therapy and the occurrence of partial necrosis was also statistically analyzed.

\section{Results}

The patients in group A, at an average of 9.7 days, required a slightly longer treatment from the initial skin suture to completion of treatment compared to the average of 6.2 days for group B patients.

Ten patients (34.5\%) from group A and four (13.8\%) from group $B$ manifested findings of partial necrosis while in inpatient care. As for the age groupings of the patients in group A, three patients between ages 18 and 40 years, five patients between 41 and 60 years, and two between ages 61 and 80 years manifested partial necrosis of wounds. Using SPSS 26.0 program, cross-analysis (chi-square, $\chi^{2}$ ) was carried out to determine the association between age and partial necrosis, while the Fisher test was carried out to attain strict verification of the association. The values revealed no statistically significant difference $\left(\chi^{2}=2.839, \mathrm{P}=0.234\right)$. As for group $\mathrm{B}$, one patient be- 
Table 2. Number of patients in each age class who experienced partial necrosis

\begin{tabular}{|c|c|c|c|c|c|c|c|c|c|c|}
\hline & \multicolumn{5}{|c|}{ Group A } & \multicolumn{5}{|c|}{ Group B } \\
\hline & Total & No necrosis & Partial necrosis & $x^{2}$ & P-value & Total & No necrosis & Partial necrosis & $x^{2}$ & P-value \\
\hline Age (yr) & & & & 2.839 & 0.234 & & & & 1.690 & 0.563 \\
\hline $18-40$ & 12 & 9 & 3 & & & 12 & 11 & 1 & & \\
\hline $41-60$ & 14 & 9 & 5 & & & 14 & 12 & 2 & & \\
\hline $61-80$ & 3 & 1 & 2 & & & 3 & 2 & 1 & & \\
\hline Total & $29(100)$ & $19(65.5)$ & $10(34.5)$ & & & $29(100)$ & 25 (86.2) & $4(13.8)$ & & \\
\hline
\end{tabular}

Values are presented as number or number (\%). Group A, the group of inpatients treated with simple dressings and antibiotics administration; Group B, the group of inpatients treated with simple dressings, antibiotics administration, prostaglandin $\mathrm{E} 1$ and supplemental oxygen for 3 days upon admission.

Statistically significant, $\mathrm{P}<0.05$.

Table 3. Types of additional treatment provided to patients who experienced partial necrosis

\begin{tabular}{lccc}
\hline & Total & $\begin{array}{c}\text { Debridement \& } \\
\text { suture }\end{array}$ & Local flap \\
\hline Group A & $10(34.5)$ & $6(20.7)$ & $4(13.8)$ \\
Group B & $4(13.8)$ & $3(10.3)$ & $1(3.5)$ \\
\hline
\end{tabular}

Values are presented as number (\%). Group A, the group of inpatients treated with simple dressings and antibiotics administration; Group B, the group of inpatients treated with simple dressings, antibiotics administration, prostaglandin $E 1$ and supplemental oxygen for 3 days upon admission.

tween the ages of 18 and 40 years, two patients of the age 41 to 60 years, and one patient between the ages 61 and 80 years manifested partial necrosis of wounds. The values also exhibited no statistically significant difference $\left(\chi^{2}=1.690, P=0.563\right)$, with no association of the age with partial necrosis of wounds (Table 2).

Regarding the difference between each group, 2.5 times more patients in group A experienced partial necrosis of wounds compared to patients in group B. The values revealed no statistically significant difference between patients in group A and group $B\left(\chi^{2}=3.390, P=0.066\right)$ (Table 2). Six patients $(20.7 \%)$ among 10 in group A presented with favorable outcomes for the dressing provided after completion of debridement and suture, and another four patients (13.8\%) underwent the additional procedures of the local flap, out of which one patient exhibited complete necrosis. In group B, three patients (10.3\%) among the four manifesting partial necrosis showed good outcomes from daily dressing after completion of simple debridement and suture, while one patient (3.5\%) was treated with additional surgery of local flap (Table 3, Fig. 1).

Consequently, the patients in group B, who were treated with the administration of PGE1 and supply of supplemental oxygen, exhibited less occurrence of necrosis, with a lower ra- tio of patients requiring additional treatment such as debridement and suture or local flap, thereby resulting in shorter periods of treatment.

\section{Case reports \\ Case 1}

A 64-year-old female was injured by pieces of glass that left an avulsion laceration on her forehead. As no underlying diseases were present, simple dressing was performed to initiate pertinent treatment. On the 4th day from the start of treatment, partial necrosis was observed on the sutured area, and a local advancement flap was executed after completion of debridement on the 7th day. The patient was discharged from the hospital upon total suture removal on the 5th day from completion of the flap surgery, with favorable wound healing results (Fig. 2).

\section{Case 2}

A 41-year-old male patient visited the emergency room with left ear injuries sustained by a machine while he was working in a factory. A wide skin avulsion comprising the narrow part of the flap pedicle on the front side of the upper auricular end, including part of the superior helix, was observed initially. The wounds were sutured primarily at the emergency room; however, the color of the skin appeared unfavorable. As the patient had no underlying diseases, simple dressing wound care was provided; findings of ischemic change were observed later (Fig. 3A). With further advancement of necrosis, debridement was done on the 5th day from the day of the injury and betadine (povidone-iodine) wet dressing was performed for 2 days for infection control (Fig. 3B). On the 7th day after the traumatic injury, a retro-auricular M-shaped skin flap was designed and incised for reconstruction of the wound (Fig. 3C). The retro-auricular skin flap was transposed to the anterior 

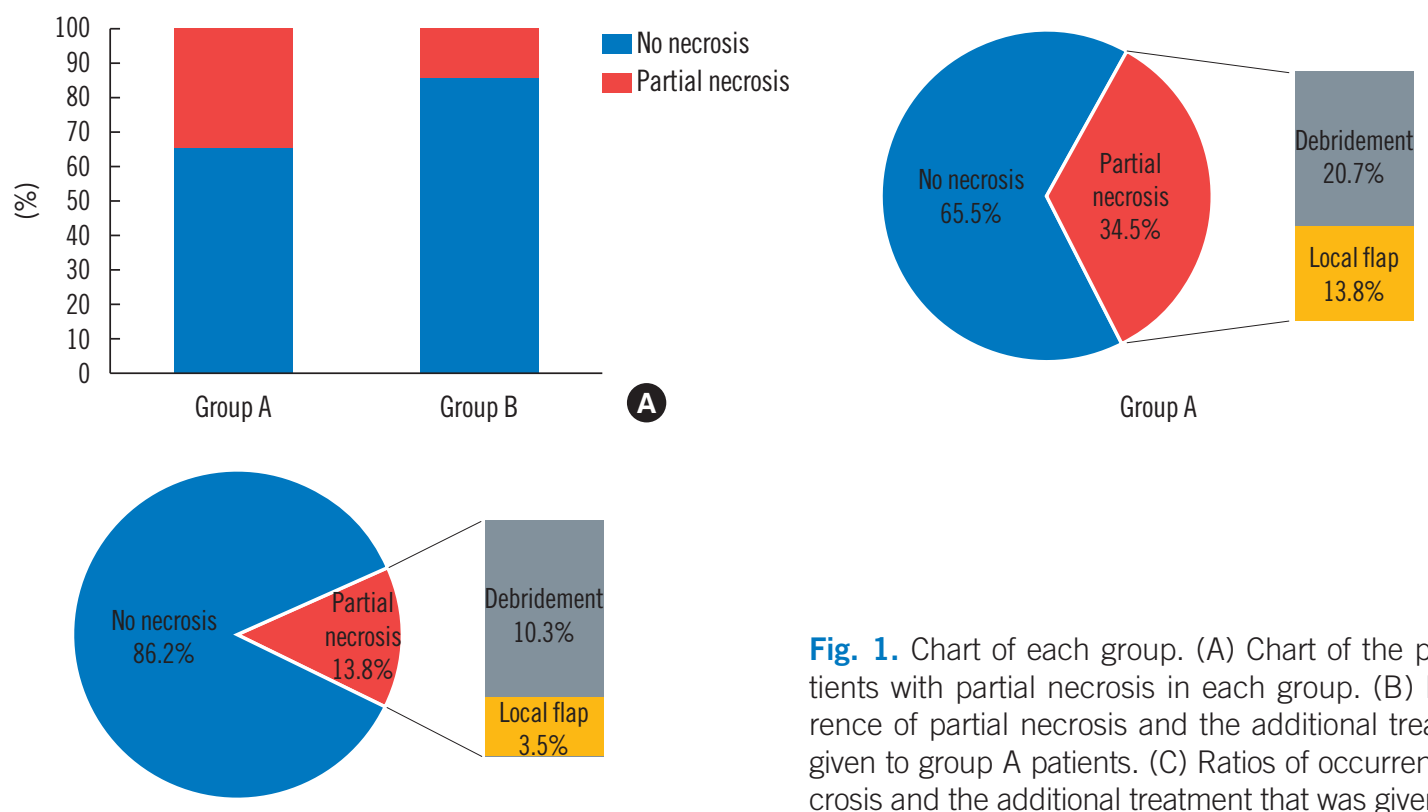

Group B

C

Fig. 1. Chart of each group. (A) Chart of the proportion of patients with partial necrosis in each group. (B) Ratios of occurrence of partial necrosis and the additional treatment that was given to group A patients. (C) Ratios of occurrence of partial necrosis and the additional treatment that was given to group B patients.
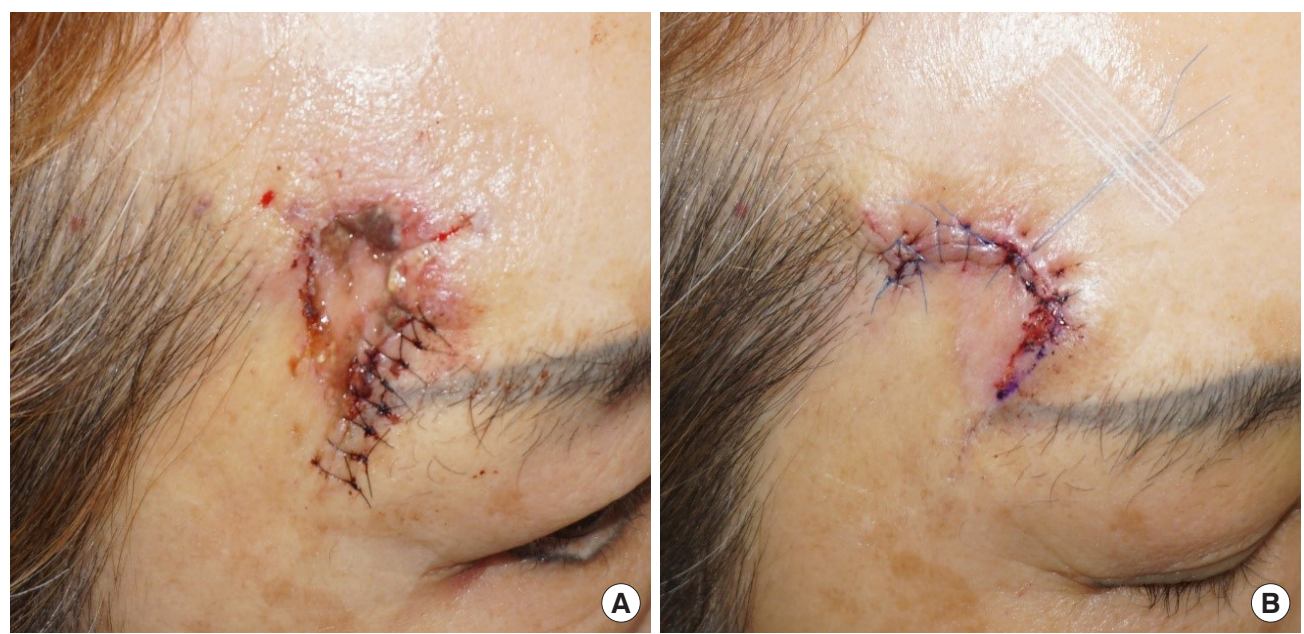

Fig. 2. Avulsion laceration caused by broken glass. (A) Wound after partial stitch removal due to ischemic changes after 4 days from skin repair. (B) Application of an advancement flap after debridement of necrotized tissue on the 7th postoperative day from the day of skin suture.

skin defect of the auricle and sutured to the wound margins of the ear (Fig. 3D). Favorable wound healing was observed, leading to the discharge of the patient from the hospital on the 7 th day from the execution of flap surgery.

\section{Case 3}

A 49-year-old male patient fell from a height, and half of the upper part of his left ear was separated from the temporal region, with the edge of separated tissue manifesting ischemic changes when the patient arrived at the emergency room. The patient was treated by administration of PGE1 and also supplemental oxygen after initial skin sutures, which led to the recovery of normal color of the ischemic tissue. No additional specific findings were observed, and the patient was discharged from the hospital on the 5th day of admission (Fig. 4).

\section{Discussion}

Skin wounds caused by injuries frequently affect subcutaneous connective tissue and microcirculation as well as the epider- 

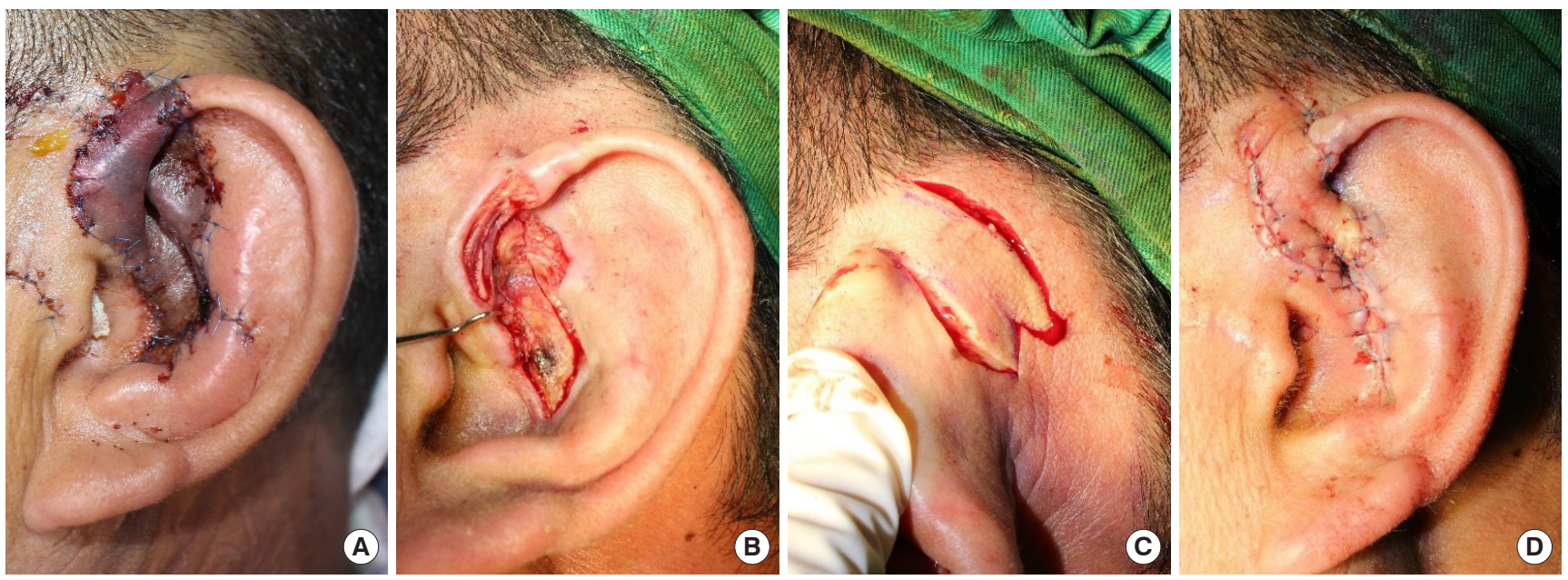

Fig. 3. Avulsion laceration caused by a factory machine. (A) Worsening ischemic changes after 3 days from skin suture. (B) Betadine wet dressings applied to the infected wound after removal of necrotized tissue. (C) Retro-auricular M-shape flap designed and incised for wound reconstruction on the 7th day from skin suture. (D) Wound healed by retro-auricular flap transposition to the pre-auricular defect.
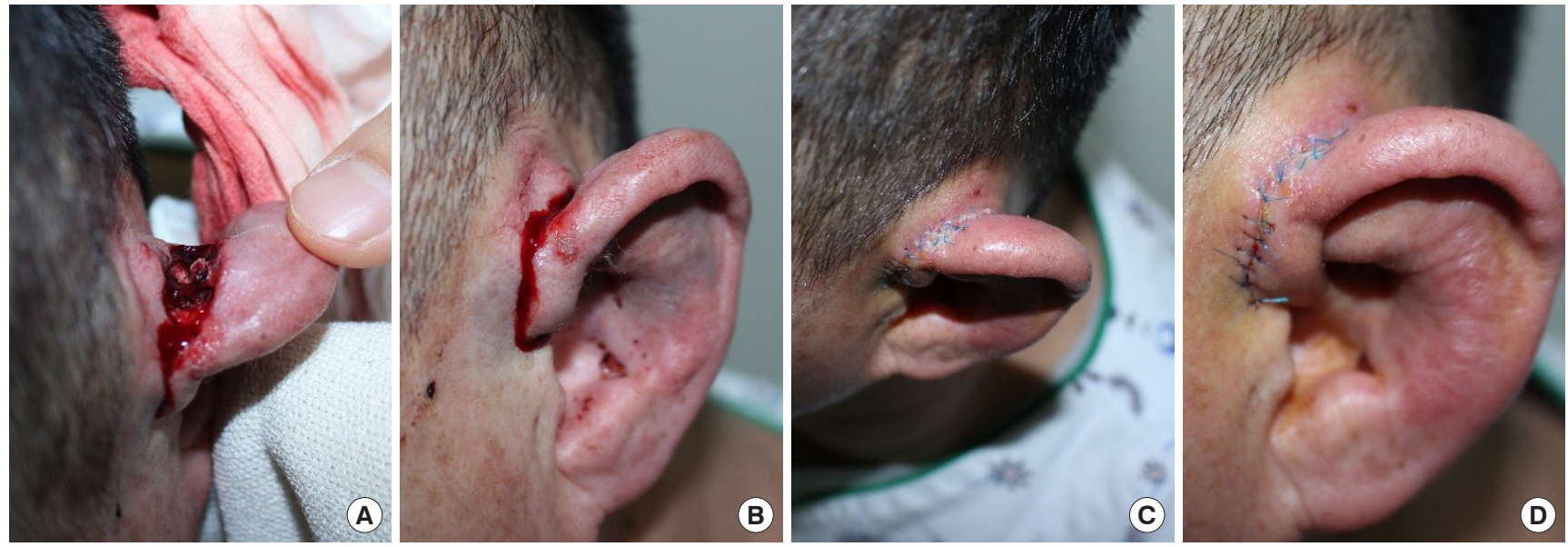

Fig. 4. Laceration of the upper left ear caused by a fall. (A, B) Half of the upper part of the left ear was separated from the temporal region and displayed ischemic color changes. (C, D) Wound on the 3rd day of prostaglandin E1 and supplemental oxygen therapy. As the patient exhibited no other problems, he was discharged after 5 days from treatment upon completion of total stitch-out.

mis and dermis. The wounded parts then undergo several steps of innate recovery; depending on the degree and depth of each wound, a recovery period from a few days to weeks are needed to complete wound healing. Physiological wound healing typically undergoes the following stages: the inflammatory phase, proliferative phase (neoangiogenesis, tissue formation, re-epithelization), and tissue remodeling phase [6,7].

Oxygen needs to be supplied to each stage of wound healing; the speed of recovery and prognoses depends on the degree of oxygen supply [8]. Oxygen is used by the mitochondria in the cells to produce adenosine triphosphate that is required for cell metabolism and synthesis of proteins, etc. in normal cells [5]. Injured tissue typically exhibits constriction of vessels and blood coagulation by platelets, thereby resulting in the formation of the platelet plug. This stage generally would last for a few minutes from tissue injury initiation. By such vascular reactions, diverse kinds of chemokines (prostaglandins, histamine, cytokine, serotonins, 5-hydroxytryptamine, leukotriene) including cytokines are secreted to initiate inflammatory reactions [9]. At the initial stage of the inflammatory reaction, oxygen is used essentially to produce reactive oxygen species (ROS; $\mathrm{H}_{2} \mathrm{O}_{2}$, and $\mathrm{O}_{2}$ ) through nicotinamide adenine dinucleotide phosphate-linked oxidase, which is critical for the oxidative killing of bacteria. Going further, ROS promotes 
the secretion of growth factors during the proliferative and tissue remodeling phases following the inflammatory phase [10].

Along with the increasing amount of oxygen in cells at stages of initial vascular disruption and vasoconstriction and resulting active inflammatory reaction and proliferation as mentioned before, a hypoxic microenvironment is created around the wound, thereby increasing the oxygen demand $[11,12]$. The supply of a sufficient amount of oxygen is mandatory for the sustainment and progress of subsequent recovery and healing stages; otherwise, the probability of necrosis would increase as the wound would progress into a chronic state $[13,14]$. The supply of an appropriate amount of oxygen to wounds depends on $\mathrm{pO}_{2}$ in the adjacent tissue and the circulating blood [15]. Methods to supply oxygen to patients can be broadly categorized into two; hyperbaric oxygen therapy and topical oxygen therapy [16]. Both methods require pertinent equipment and facilities, and in particular, hyperbaric oxygen therapy entails a risk of oxygen toxicity. Therefore, we employed supplemental oxygen therapy by intranasal cannula rather than hyperbaric oxygen therapy [11].

Part of the inpatients who received skin sutures for avulsion wounds at the emergency room from March 2017 to December 2018 exhibited necrotized wounds; it was hypothesized that the cause was insufficient blood supply of the wound. In cases of multiple deep lacerations, avulsion wounds from crushing injuries, or wounds reconstructed with local flaps, the tension remaining in each skin margin resulted in poor blood supply. As a means to cope with this problem, from January 2019, patients who needed inpatient care were administered PGE1 and supplemental oxygen during the first 3 days of admission, which appeared to decrease the frequency and degree of necrosis. PGE1 relaxes thin blood vessels and increases blood flow, which helps with peripheral circulation $[3,4]$. PGE1 may have increased the supply of immune substances and nutrients to wounds through expanded blood vessels that had previously been constricted, and the supplemental oxygen that fulfilled the demand for the oxygen needed for the wound healing resulted in reduced flap necrosis.

In conclusion, ischemic wounds displayed signs of improved healing in patients who were treated with administration of PGE1 and supplemental oxygen through a nasal cannula. Although there were no statistically significant differences, the authors of this study believe that this was because the sample size was not large. Even after the conclusion of this study, the authors continue to use PGE1 and supplemental oxygen for trauma inpatients. In addition, the same treatment is also being applied to patients who have received flap surgery for skin defects and pressure sores, to be discussed in a followup study. We expect such research on post-operational care will help secondary or smaller hospitals as well.

The present study has some shortcomings. The individual differences in patients such as fluid gas exchange, blood hemoglobin level, cardiac output, peripheral perfusion rate, and capillary density in wound tissue have not been considered. In addition, the absence of equipment to measure the partial pressure of oxygen in the tissue after the supply of oxygen through a nasal cannula and PGE1 needs to be addressed. However, it was identified that the treatment helped the wound healing of inpatients who were hospitalized due to poor blood circulation after flap procedures or those who suffered from lacerations, particularly avulsion wounds. The present paper suggested the clinical significance of employing an additional treatment for a relatively reduced ratio of progression to necrosis in wounds.

\section{Conflict of interest}

No potential conflict of interest relevant to this article was reported.

\section{ORCID iDs}

Bongsoo Baik https://orcid.org/0000-0002-8019-3310

Sulki Park https://orcid.org/0000-0003-0243-2790

Soyoung Ji https://orcid.org/0000-0002-6060-1452

Wansuk Yang https://orcid.org/0000-0002-2706-5678

Junekey Lee https://orcid.org/0000-0002-5343-2806

\section{References}

1. Kuwahara H, Fazhi Q, Sugihara T, et al. The effects of lipoprostaglandin E1 on axial pattern flaps in rabbits. Ann Plast Surg 1995;35:620-6.

2. Himmelreich G, Hundt K, Neuhaus P, et al. Evidence that intraoperative prostaglandin E1 infusion reduces impaired platelet aggregation after reperfusion in orthotopic liver transplantation. Transplantation 1993;55:819-26.

3. Lee MK, Cho SW, Chang H, et al. The effect of prostaglandin E1 on the resolution of ecchymosis. J Wound Manag Res 2018;14:44-8.

4. Tonseth KA, Sneistrup C, Berg TM. Prostaglandin E1 increases microcirculation in random pattern flaps on rats 
measured with laser doppler perfusion imaging. Plast Reconstr Surg Glob Open 2017;5:e1202.

5. Gordillo GM, Sen CK. Revisiting the essential role of oxygen in wound healing. Am J Surg 2003;186:259-63.

6. Gurtner GC, Werner S, Barrandon Y, et al. Wound repair and regeneration. Nature 2008;453:314-21.

7. Singer AJ, Clark RA. Cutaneous wound healing. N Engl J Med 1999;341:738-46.

8. Tandara AA, Mustoe TA. Oxygen in wound healing: more than a nutrient. World J Surg 2004;28:294-300.

9. Yoon HS, Na YC, Choi KH, et al. Wound healing effect of regenerated oxidized cellulose versus fibrin sealant patch: an in vivo study. Arch Craniofac Surg 2019;20:289-96.

10. Gottrup F. Oxygen in wound healing and infection. World J Surg 2004;28:312-5.
11. Jonsson K, Jensen JA, Goodson WH 3rd, et al. Tissue oxygenation, anemia, and perfusion in relation to wound healing in surgical patients. Ann Surg 1991;214:605-13.

12. Lazarus GS, Cooper DM, Knighton DR, et al. Definitions and guidelines for assessment of wounds and evaluation of healing. Wound Repair Regen 1994;2:165-70.

13. Janis JE, Harrison B. Wound healing: part I. Basic science. Plast Reconstr Surg 2014;133:199e-207e.

14. Gottrup F. Physiology and measurement of tissue perfusion. Ann Chir Gynaecol 1994;83:183-9.

15. Kalliainen LK, Gordillo GM, Schlanger R, et al. Topical oxygen as an adjunct to wound healing: a clinical case series. Pathophysiology 2003;9:81-7.

16. Broussard CL. Hyperbaric oxygenation and wound healing. J Vasc Nurs 2004;22:42-8. 